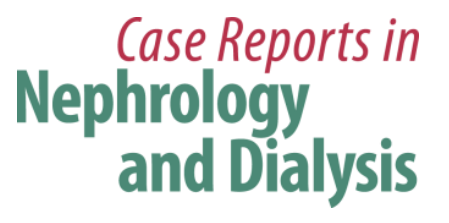

Case Rep Nephrol Dial 2017;7:18-25

DOI: 10.1159/000457950

Published online: March 2, 2017

2017 The Author(s)Published by S.

Karger AG, Basel

www.karger.com/cnd

This article is licensed under the Creative Commons Attribution-NonCommercial 4.0 International License (CC BY-NC) (http://www.karger.com/Services/OpenAccessLicense) Usage and distribution for commercial purposes requires written permission.

\title{
Discontinuation of Peritoneal Dialysis after Late Initiation of Eculizumab in a Case of Familial Atypical Hemolytic-Uremic Syndrome: A Case Report
}

\author{
Rafael Alonso Valente Giannina Elena García Rodríguez \\ Yanina García Marcote Manuel Fidalgo Díaz Vanesa Becerra Mosquera \\ Daniel Novoa García Teresa Cordal Martínez Cándido Díaz Rodríguez
}

Nephrology Department, Hospital Clínico Universitario de Santiago de Compostela,

Santiago de Compostela, Spain

\section{Keywords}

Atypical hemolytic-uremic syndrome $\cdot$ Eculizumab $\cdot$ Mutation $\cdot$ Complement inactivating agents

\section{Abstract}

Background: Atypical hemolytic-uremic syndrome is caused by a thrombotic microangiopathy and manifests itself with hemolytic anemia, thrombocytopenia, and organ ischemia. Its etiology is a mutation affecting the genes encoding for proteins of the complement system. Early treatment with eculizumab (8.6 months from the moment of presentation), a humanized monoclonal antibody against complement, is shown to be effective in controlling symptoms and reversing organ damage. We present a patient with a mutation not previously described in the literature. Late treatment with eculizumab resulted in a good therapeutic re- 


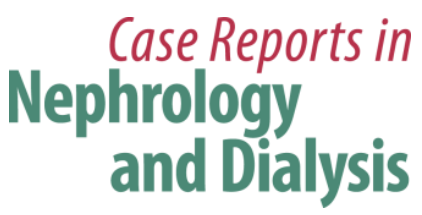

Case Rep Nephrol Dial 2017;7:18-25

sponse, eliminating the need for peritoneal dialysis. Case Presentation: A 34-year-old woman showed symptoms and laboratory findings consistent with atypical hemolytic-uremic syndrome. Genetic analysis revealed an unusual mutation of the complement regulatory gene not seen previously. Due to unavailability of eculizumab at the time of presentation, conventional treatment was started with poor response. Late initiation of eculizumab resulted in discontinuation of peritoneal dialysis and yielded a good and sustained clinical response. Conclusions: This case shows that eculizumab treatment for patients with atypical hemolyticuremic syndrome, even when initiated many months after beginning on dialysis, might offer substantial benefits and improve the patients' quality of life.

(C) 2017 The Author(s)

Published by S. Karger AG, Basel

\section{Background}

Hemolytic-uremic syndrome (HUS) is a life-threatening condition caused by a thrombotic microangiopathy producing microangiopathic hemolytic anemia, thrombocytopenia, and organ ischemia. HUS mainly affects the kidneys, ultimately leading to renal failure [1]. HUS is commonly caused by infection with a Shiga-like toxin-producing strain of Escherichia coli. By contrast, atypical HUS (aHUS) occurs as a consequence of mutations in genes that encode various proteins involved in the alternative pathway of the complement system. This subtype accounts for $10 \%$ of all cases of HUS [2].

Traditionally, plasma exchange and plasma infusion had been the first line of treatment for patients with aHUS. Despite some controversy, expert opinion postulated that plasmapheresis could be effective, especially in patients with a mutation in complement factor $\mathrm{H}$ (CFH), and possibly in patients with mutations in complement factor I (CFI), factor B (CFB), or C3 as well. No beneficial effect of plasmapheresis is expected in patients with a mutation in the membrane cofactor protein (MCP). Plasmapheresis is important for removing antibodies from the serum of patients with aHUS that have anti-CFH autoantibodies, although in such cases, the production of these autoantibodies should also be suppressed by treatment with additional immunosuppressive agents. Plasmapheresis should be started as soon as the diagnosis of aHUS is made $[3,4]$. However, within 1 year following diagnosis of aHUS, up to $65 \%$ of patients treated with plasma exchange or plasma infusion sustain permanent renal damage, progress to end-stage renal disease (ESRD), or succumb to their illness. Additionally, $60-90 \%$ of patients with aHUS who underwent kidney transplantation suffer of graft failure in the first year [4].

A biologic explanation of the high rate of disease progression and mortality could be that these traditional treatments might transiently maintain normal levels of hematologic parameters, but they do not cure the underlying systemic disease [3]. Eculizumab is a monoclonal antibody against complement component C5 that is designed to suppress terminal complement activation and to prevent thrombotic microangiopathy. In prospective clinical trials, eculizumab has been shown to inhibit thrombotic microangiopathy progression and to prevent or even reverse organ damage in patients with aHUS $[5,6]$. In these trials, the median interval between the clinical manifestations of aHUS and the initiation of treatment with eculizumab was approximately 9 months, yielding good results. Thus, the most recent guidelines recommend initiation of eculizumab treatment at the moment of diagnosis. However, 


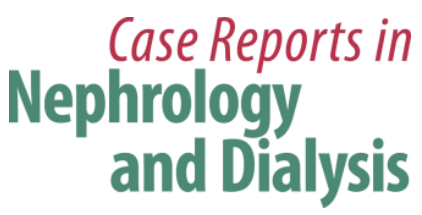

Case Rep Nephrol Dial 2017;7:18-25
$\begin{array}{ll}\text { DOI: } 10.1159 / 000457950 & \begin{array}{l}\text { (C) } 2017 \text { The Author(s). Published by S. Karger AG, Basel } \\ \text { www.karger.com/cnd }\end{array}\end{array}$

Alonso Valente et al.: Discontinuation of Peritoneal Dialysis after Late Initiation of Eculizumab in a Case of Familial Atypical Hemolytic-Uremic Syndrome: A Case Report

the duration of eculizumab treatment remains a controversial issue [6]. Moreover, data regarding clinical outcomes among patients starting eculizumab treatment long after the acute clinical manifestations of aHUS are scarce $[7,8]$.

Here, we describe the case of a 34-year-old woman with CFH gene abnormalities, who developed aHUS with severe renal impairment that progressed to ESRD requiring treatment with peritoneal dialysis. Treatment with eculizumab was started 62 months after the initial manifestation of aHUS. Previously, the patient had been treated with dialysis, due to unavailability of the drug at diagnosis, the presence refractory hypertension despite treatment with high doses of 6 anti-hypertensive drugs, and the quality of her 24-hour urine. Eculizumab treatment resulted in improvements on platelet levels, arterial blood pressure control, and renal function. These improvements resulted in peritoneal dialysis discontinuation. Additionally, a genetic analysis of the $\mathrm{CFH}$ gene revealed the presence of a mutation not previously described in the literature.

\section{Case Presentation}

A 34-year-old woman with an unremarkable past medical and family history presented at the emergency room with symptoms of nausea, abdominal discomfort, and pallor for 7 days. High blood pressure levels were recorded all days. She had previously received nonsteroidal anti-inflammatory drug treatment for toothache for 3 days. There was no recent history of diarrhea, dysentery-like illness, pregnancy, miscarriage, or use of contraceptives.

Physical examination of the patient revealed pallor of the skin and mucous membranes. Her blood pressure was 155/100 mm Hg. Otherwise, physical examination was unremarkable.

Laboratory tests were indicative of microangiopathic hemolytic anemia with hemoglobin levels of $9.4 \mathrm{~g} / \mathrm{dL}$ (normal range, 12.2-16.1 g/dL). Peripheral blood smear revealed anisopoikilocytosis (red blood cells of varying shapes and sizes), including the presence of fragmented red blood cells (schistocytes and helmet cells). Coomb's test was negative, and haptoglobin was absent. The platelet count was $27,000 / \mathrm{mm}^{3}$. Renal damage was present, with serum creatinine levels of $5.8 \mathrm{mg} / \mathrm{dL}$ (normal range, $0.43-0.9 \mathrm{mg} / \mathrm{dL}$ ), urea levels of $173 \mathrm{mg} / \mathrm{dL}$, phosphorus levels of $6.4 \mathrm{mg} / \mathrm{dL}$, and lactate dehydrogenase levels of 1,394 UI/L. There were no further pathological biochemical parameters. Urinalysis revealed 3+ albumin, and microscopy of the urine sediment yielded 5-7 red cells per X power field. Prothrombin time was $12 \mathrm{~s}$, and activated partial thromboplastin time was $26.5 \mathrm{~s}$. Antinuclear antibodies, antiphospholipid antibodies, rheumatoid factor, anti-neutrophil cytoplasmic antibody, and anti-native DNA antibodies were not detected. Blood and urine cultures were sterile. The patient's serum tested negative for hepatitis B virus, hepatitis $C$ virus, and human immunodeficiency virus. A hyperechogenic cortex was displayed on the renal ultrasound scan. The exploration was otherwise normal. Renal biopsy was not performed.

After ruling out secondary causes of thrombotic microangiopathy, a definitive diagnosis of aHUS was made. Plasma exchange was initiated with the patient receiving exchanges twice daily, for a total of 31 sessions. However, her renal function did not improve, and hemodialysis therapy was subsequently initiated. 


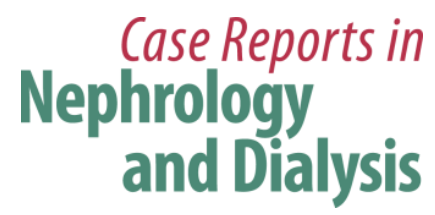

Case Rep Nephrol Dial 2017;7:18-25

DOI: $10.1159 / 000457950$

2017 The Author(s). Published by S. Karger AG, Base www.karger.com/cnd

Alonso Valente et al.: Discontinuation of Peritoneal Dialysis after Late Initiation of Eculizumab in a Case of Familial Atypical Hemolytic-Uremic Syndrome: A Case Report

A further diagnostic workup was performed. ADAMTS13 activity in the plasma was $86.5 \%$ (normal range, $40-124 \%$ ) and thus, it was found to be normal. The plasma concentrations of complement components were as follows: C3, $73.6 \mathrm{mg} / \mathrm{dL}$ (normal range, 77-210 $\mathrm{mg} / \mathrm{dL}$ ), C4, $28.4 \mathrm{~g} / \mathrm{L}$ (normal range, 14-47 g/L), and CFH, $21.37 \mathrm{mg} / \mathrm{dL}$ (normal range, 12$56 \mathrm{mg} / \mathrm{dL}$ ). CFI activity was 85\% (normal range, 71-115\%), MCP was 117\% (normal range, 91-109\%), and anti-CFH antibodies were absent. Mutation screening of CFH, CFI, CD46, C3, and CFB was conducted. This analysis revealed a heterozygous single-nucleotide mutation in the exon 13 of the CFH gene (c.1707>A; Cys569stop) that was predicted to truncate the protein product by introducing a stop codon (Cys569stop). This mutation was not defined in the Exome Sequencing Project. No mutations were found either for CFI or MCP genes. Moreover, the patient carries the $C F H$ and $M C P$ risk haplotypes for aHUS in heterozygous pattern.

The patient was switched to automated peritoneal dialysis (APD) after almost 2 months of hemodialysis. Her APD schedule consisted of 5 overnight exchanges of $2 \mathrm{~L}$ and 1 icodextrin (extraneal) wet day. At this moment, the patient's residual renal function (RRF) was 3-4 mL/min, and her urine output was $700 \mathrm{~mL}$ per day (urea clearance of $1,400 \mathrm{mg} / \mathrm{dL}$ and creatinine clearance of $108 \mathrm{mg} / \mathrm{dL}$ ). Blood pressure remained poorly controlled $(>163 / 105$ $\mathrm{mm} \mathrm{Hg}$ ) despite administration of maximal doses of 6 different antihypertensive drugs. Platelet count was $103,000 / \mathrm{mm}^{3}$, and hemoglobin level was $12.2 \mathrm{~g} / \mathrm{dL}$.

Sixty-two months following diagnosis and treatment with dialysis, the patient remained on APD with sustained high blood pressure, anemia and thrombocytopenia. At this point, she was re-evaluated, as her clinical symptoms and hematological and biochemical profile remained poorly controlled. Treatment with complement-blocking therapy with eculizumab was initiated. At the time of initiation of eculizumab, the patient was asymptomatic and in good clinical status. Blood pressure values remained 160/105 mm Hg despite the use of full doses of 6 hypotensive drugs (verapamil, trandolapril, atenolol, doxazosin, aliskiren, and furosemide). Platelet count was $103,000 / \mathrm{mm}^{3}$. Diuresis was $700 \mathrm{~mL}$ with proteinuria of 0.3 $\mathrm{g} / \mathrm{L}$ and creatinine of $7.53 \mathrm{mg} / \mathrm{dL}$. RRF was $3.9 \mathrm{~mL} / \mathrm{min}$ (urea clearance of $2.2 \mathrm{~mL} / \mathrm{min}$ and creatinine clearance of $5.6 \mathrm{~mL} / \mathrm{min}$ ). The patient was on continuous cycling peritoneal dialysis with $10 \mathrm{~L}$ overnight exchanges and $2 \mathrm{~L}$ of icodextrin during the day. In order to prevent meningococcal infection, the patient received prophylactic antibiotic treatment with ciprofloxacin and vaccination against Neisseria meningitides before receiving the first dose of eculizumab on August 5, 2013. Follow-up was performed every 3 months (Table 1). The patient's clinical condition improved within 6 months of starting eculizumab therapy: RRF increased to $11 \mathrm{~mL} / \mathrm{min}$ and urine output increased to 2,000 $\mathrm{mL}$ per day. Levels of hemoglobin, platelets, and lactate dehydrogenase returned to normal ranges. The patient's blood pressure was better controlled with 4 drugs (furosemide, verapamil, trandolapril, and metoprolol), with levels below 120/70 mm Hg (Table 1). Improvement was sustained through successive follow-up visits. Fifteen months after the initiation of eculizumab, kidney creatinine clearance rate was $30 \mathrm{~mL} / \mathrm{min}$, RRF was $20 \mathrm{~mL} / \mathrm{min}$, and urine output was 2,300 $\mathrm{mL}$ per day. At 16 months of follow-up, APD dose was reduced to 3 overnight exchanges of 2 L, 5 days per week, and extraneal exchange and wet day were no longer required. Currently, dialysis has been successfully discontinued.

Treatment with eculizumab was well tolerated by the patient throughout the follow-up period. At present, and in anticipation of her progress in the next months, we are evaluating 


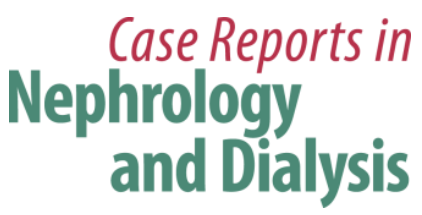

Case Rep Nephrol Dial 2017;7:18-25
\begin{tabular}{l|l}
\hline DOI: $10.1159 / 000457950$ & $\begin{array}{l}\text { (c) } 2017 \text { The Author(s). Published by S. Karger AG, Basel } \\
\text { www.karger.com/cnd }\end{array}$
\end{tabular}

Alonso Valente et al.: Discontinuation of Peritoneal Dialysis after Late Initiation of Eculizumab in a Case of Familial Atypical Hemolytic-Uremic Syndrome: A Case Report

the options of either keeping the patient under supervision for advanced renal disease or including her in the renal transplant waiting list.

\section{Discussion}

The present case study outlines the clinical management of a patient with aHUS carrying a novel single-nucleotide mutation in $\mathrm{CFH}$ who did not respond to traditional treatment options. Subsequent treatment with eculizumab - a specific inhibitor of the complement pathway - led to a marked improvement in renal function, which in turn lowered the blood pressure and the requirements of APD.

aHUS is a rare thrombotic microangiopathy caused by genetic defects in the complement system regulation and is associated with a very poor prognosis. Mortality approaches $25 \%$ during its acute phase, and ESRD develops in nearly half of the patients within 1 year of diagnosis [2]. Traditionally, plasma exchange and plasma infusion were the standard treatment for aHUS. However, many patients did not respond to plasma therapy, and some required prolonged therapy because these treatments do not correct the underlying complement deregulation [2].

More recently, the introduction of complement inhibitor eculizumab has been a breakthrough in treatment of aHUS, dramatically improving the patients outcome. Eculizumab is a humanized monoclonal antibody that inhibits complement function by binding with high affinity to the human C5 complement protein and blocks the formation of proinflammatory C5a and membrane-attack complex C5b-9 [5, 6]. In prospective clinical trials in patients with aHUS, early intervention with eculizumab was associated with significant improvement in estimated GFR $[5,6]$. However, the mean time between the onset of symptoms and the initiation of eculizumab treatment in clinical trials is shorter [3].

The median interval between clinical presentation of aHUS and initiation of eculizumab treatment ranged between 0.8 and 8.6 months among patients with progressive thrombotic microangiopathy and renal damage (regardless of decreases in platelet counts). Initiation of eculizumab treatment after long-term dialysis is a rare occurrence but it has been previously reported to improve renal function $[7,8]$. In the present study, the patient initiated eculizumab treatment after an interval of 62 months (since the drug was not available at diagnosis). Despite this lag, substantial improvements were noted in multiple clinical and biochemical parameters. This suggests that eculizumab therapy initiation can be effective beyond the previously reported upper limit of 8.6 months. During treatment, there was no hemolytic crisis although platelet levels were not optimal (Table 1).

Mutations have been identified in approximately $50-70 \%$ of patients with aHUS. These mutations affect both genes encoding complement regulators (CFH, CFI, CFH-related proteins, and MCP) and those encoding complement activators (CFB and C3) [2]. Three DNA sequence analyses of $C F H, M C P$, and $C F I$ genes from our patient revealed the presence of a heterozygous mutation in exon 13 of the $C F H$ gene. This mutation results in a cysteine residue being replaced by a stop codon at position 569 within the SCR10 domain of the CFH protein. Consequently, this premature stop codon results in the production of a truncated protein that lacks the $\mathrm{C}$-terminal portion (the factor $\mathrm{H}$ region), which is crucial for the development of aHUS. Characterization of CFH in the plasma of the patient by Western blot analy- 


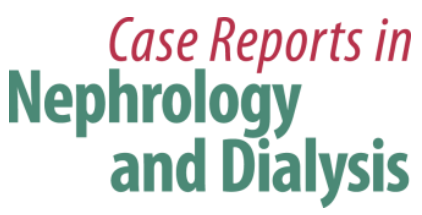

Case Rep Nephrol Dial 2017;7:18-25
$\begin{array}{ll}\text { DOI: } 10.1159 / 000457950 & \begin{array}{l}\text { (C) } 2017 \text { The Author(s). Published by S. Karger AG, Basel } \\ \text { www.karger.com/cnd }\end{array}\end{array}$

Alonso Valente et al.: Discontinuation of Peritoneal Dialysis after Late Initiation of Eculizumab in a Case of Familial Atypical Hemolytic-Uremic Syndrome: A Case Report

sis suggested that, unlike wild-type $\mathrm{CFH}$, the truncated protein was not secreted into the plasma, causing a partial deficiency of factor $\mathrm{H}$. This deficit was most likely responsible for the slight activation in the alternative complement pathway (decreased C3 levels and C4 levels up to the lower limits of normal range). This was observed during the initial biochemical evaluation of the patient. This $C F H$ mutation has not been previously described in the literature to have pathogenic implications and has not been clearly associated with genetic susceptibility in the pathogenesis of aHUS. This genetic change aside, the patient also carried risk haplotypes for aHUS for the CFH and MCP heterozygous genes.

Mutations in complement regulatory genes can also be observed in healthy family members. In the present case, the patient's mother never developed the disease, although she has the same $C F H$ mutation and risk haplotypes. The penetrance of aHUS among carriers of mutations in $C F H, C F I$, and $M C P$ is approximately $50-60 \%$. Therefore, although genetic aberrations are important, they are not always sufficient for the development of aHUS. Affected patients have been reported to have mutations in more than one gene, in combination with aHUS risk haplotypes for CFH and MCP genes.

Eculizumab has previously shown its effectiveness in preventing disease recurrence after renal transplantation [9]. Before treatment with eculizumab, renal transplant was contraindicated for our patient due to the presence of a mutation with a high rate of recurrence [10]. However, the improvement of renal function was sufficient to allow dialysis discontinuation and minimizing the need for renal transplant. Because we did not perform a renal biopsy at presentation, there is no kidney anatomopathological data, which could determine the degree of renal injury. Moreover, we cannot predict whether the observed improvements in renal function and blood pressure (sustained for 15 months) will be maintained or will continue to improve sufficiently, making the withdrawal of dialysis a permanent occurrence. There is no previous evidence about the safety of eculizumab suspension. Thus, the patient is still currently under treatment. Further studies are needed to support a larger dose range or even its suspension. However, even if improvements in renal function were not sufficient to allow for a definitive and sustained withdrawal of dialysis, renal transplant remains an option for this patient in the future.

\section{Conclusion}

Treatment of familial aHUS with eculizumab - even when it is initiated many months after the beginning of dialysis - might offer substantial benefits in terms of renal function, blood pressure control and morbidity. Such improvements are likely to improve the quality of life for such patients. A limitation of the present study is that the results cannot be applied to all patients with aHUS because etiological factors, other than complement activation, might be involved. Moreover, these results are derived from an isolated case of a pathological entity with a very low incidence [11]. Surrogate markers are needed to be able to identify patients who will exhibit a treatment response. In addition, it is essential to further investigate the most appropriate dose of eculizumab, dosage intervals, and duration of treatment to decrease the high costs associated with the use of this therapy. Prospective studies of eculizumab in large cohorts of patients with long duration of aHUS are now required to validate the applicability of the positive experience described in this case study. 


\section{Acknowledgements}

The authors thank Nature Publishing Group Iberoamérica and Beatriz Rodríguez Cubillo, MD and Pablo Rivas, MD, PhD for editing the manuscript and for editorial assistance.

\section{Statement of Ethics}

Written informed consent was obtained from the patient for publication of this case report and any accompanying images. A copy of the written consent is available for review by the Editor of this journal.

\section{Disclosure Statement}

The authors declare that they have no competing interests.

\section{Authors' Contributions}

R.A.V. conceived the article and drafted the manuscript. All authors read and approved the final manuscript.

\section{References}

1 Shenkman B, Einav Y: Thrombotic thrombocytopenic purpura and other thrombotic microangiopathic hemolytic anemias: diagnosis and classification. Autoimmun Rev 2014;13:584-586.

Nayer A, Asif A: Atypical hemolytic-uremic syndrome: a clinical review. Am J Ther 2016;23:e151-e158. Sinha A, Gulati A, Saini S, et al: Prompt plasma exchanges and immunosuppressive treatment improves the outcomes of anti-factor $\mathrm{H}$ autoantibody-associated hemolytic uremic syndrome in children. Kidney Int 2014;85:1151-1160.

- Verhave JC, Wetzels JF, van de Kar NC: Novel aspects of atypical haemolytic uraemic syndrome and the role of eculizumab. Nephrol Dial Transplant 2014;29(suppl 4):iv131-iv141.

5 Legendre CM, Licht C, Muus P, et al: Terminal complement inhibitor eculizumab in atypical hemolyticuremic syndrome. New Engl J Med 2013;368:2169-2181.

6 Fakhouri F, Fila M, Provot F, et al: Pathogenic variants in complement genes and risk of atypical hemolytic uremic syndrome relapse after eculizumab discontinuation. Clin J Am Soc Nephrol 2017;12:50-59.

7 Povey H, Vundru R, Junglee N, Jibani M: Renal recovery with eculizumab in atypical hemolytic uremic syndrome following prolonged dialysis. Clin Nephrol 2014;82:326-331.

8 Emirova K, Volokhina E, Tolstova E, van den Heuvel B: Recovery of renal function after long-term dialysis and resolution of cardiomyopathy in a patient with aHUS receiving eculizumab. BMJ Case Rep 2016, DOI: 10.1136/bcr-2015-213928.

-9 Matar D, Naqvi F, Racusen LC, Carter-Monroe N, Montgomery RA, Alachkar N: Atypical hemolytic uremic syndrome recurrence after kidney transplantation. Transplantation 2014;98:1205-1212.

10 Saland JM, Ruggenenti P, Remuzzi G: Liver-kidney transplantation to cure atypical hemolytic uremic syndrome. J Am Soc Nephrol 2009;20:940-949. 


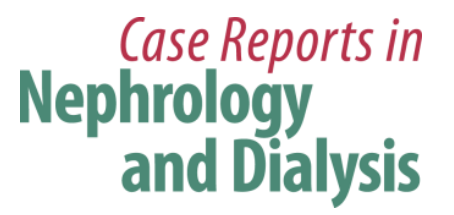

Case Rep Nephrol Dial 2017:7:18-25

(C) 2017 The Author(s). Published by S. Karger AG, Basel www.karger.com/cnd

Alonso Valente et al.: Discontinuation of Peritoneal Dialysis after Late Initiation of Eculizumab in a Case of Familial Atypical Hemolytic-Uremic Syndrome: A Case Report

11 Le Quintrec M, Zuber J, Moulin B, et al: Complement genes strongly predict recurrence and graft outcome in adult renal transplant recipients with atypical hemolytic and uremic syndrome. Am J Transplant 2013;13:663-675.

Table 1. Response of the patient to the treatment with eculizumab for a period of 15 months

\begin{tabular}{|c|c|c|c|c|c|c|c|}
\hline Time point & $\begin{array}{l}\mathrm{BP}, \\
\mathrm{mm} \mathrm{Hg}\end{array}$ & $\begin{array}{l}\text { Platelets, } \\
\times 1,000 / \mu \mathrm{L}\end{array}$ & $\begin{array}{l}\text { Creatinine, } \\
\mathrm{mg} / \mathrm{dL}\end{array}$ & $\begin{array}{l}\mathrm{RRF} \\
\mathrm{mL} / \mathrm{min}\end{array}$ & $\begin{array}{l}\text { Diuresis, } \\
\mathrm{mL}\end{array}$ & BPD & $\begin{array}{l}\text { Weekly } \\
\text { KT/V/CCr }\end{array}$ \\
\hline Pretreatment & $163 / 105$ & 103.000 & 7.53 & 3.9 & 700 & $6^{*}$ & $2.24 / 59.5$ \\
\hline 3 months & $115 / 76$ & 107.000 & 5.85 & 7.5 & 1,500 & $5^{* *}$ & $2.74 / 64$ \\
\hline 6 months & $103 / 56$ & 153.000 & 4.75 & 11 & 2,000 & $5^{* *}$ & $2.67 / 110.2$ \\
\hline 9 months & $109 / 68$ & 125.000 & 4.14 & 13 & 1,800 & 4 & $2.67 / 113$ \\
\hline 12 months & $115 / 76$ & 114.000 & 4.15 & 14 & 2,200 & 3 & $2.98 / 157.7$ \\
\hline 15 months & $110 / 72$ & 182.000 & 3.87 & 30 & 2,400 & 2 & $3.11 / 191$ \\
\hline
\end{tabular}

BP, blood pressure; BPD, number of BP drugs administered; CCr, creatinine clearance; KT/V, urine kinetics; RRF, renal residual function (mean of urea and creatinine clearances). * Maximal doses. ** Half doses. 